Proceedings of the Edinburgh Mathematical Society (2008) 51, 765-778 (C)

DOI:10.1017/S0013091506000939 Printed in the United Kingdom

\title{
VECTOR FIELDS TANGENT TO FOLIATIONS
}

\author{
L. F. MARTINS ${ }^{1}$ AND F. TARI ${ }^{2}$ \\ ${ }^{1}$ Departamento de Matemática, Instituto de Biociências, Letras e Ciências Exatas, \\ Universidade Estadual Paulista 'Júlio de Mesquita Filho', R. Cristóvão Colombo, \\ 2265 Jardim Nazareth, CEP 15054-000, São José do Rio Preto SP, \\ Brazil (lmartins@ibilce.unesp.br) \\ ${ }^{2}$ Department of Mathematical Sciences, Durham University, Science Laboratories, \\ South Road, Durham DH1 3LE, UK (farid.tari@durham.ac.uk)
}

(Received 31 July 2006)

Abstract We investigate in this paper the topological stability of pairs $(\omega, X)$, where $\omega$ is a germ of an integrable 1-form and $X$ is a germ of a vector field tangent to the foliation determined by $\omega$.

Keywords: 1-form; foliation; singularity; topological stability; vector field

2000 Mathematics subject classification: Primary 58A10; 37C15; 32S65

\section{Introduction}

We study in this paper pairs $(\omega, X)$, where $\omega$ is a germ, at the origin in $\mathbb{R}^{n}$, of an integrable 1-form and $X$ is a germ, at the origin, of a vector field tangent to (the germ of) the foliation determined by $\omega$ (tangent to $\omega$ for short). We are, in particular, interested in the topological stability of such pairs. When $\omega=\mathrm{d} f$ for some germ of a function $f$, the vector field $X$ has $f$ as a first integral. In [4], Lara studied the local topological stability of pairs $(f, X)$, where $f$ is a function with a Morse singularity and $X$ is a vector field tangent to the level sets of $f$. A complete classification of stable pairs is given in [4] for the cases in which $n=3,4$, and for $n \geqslant 5$ if the index of the singularity of $f$ is 0 or $n$.

We study here a more general situation, namely when the foliation is given locally by a germ of an integrable 1-form, and deal with the following question: given a topologically stable pair $(\omega, X)$, is $\omega$ a topologically stable 1 -form? We prove here that this is the case for germs of analytic pairs (Theorem 3.1). The proof uses the fact that in the analytic case we can characterize all the vector fields $X$ tangent to $\omega$ in terms of $\omega$. (A similar question can be asked regarding the stability of $X$; see $\S 2$.)

We give a classification of stable pairs (Theorem 3.2) when $\omega$ has a non-degenerate singularity, thus extending the results of Lara. When the index of the singularity of $\omega$ is 2 or $n-2$, we restrict ourselves to the set of analytic 1-forms. We show in Theorem 3.4 that if $\omega$ is as above, then for any pair $(\omega, X)$ there exists a tangent pair $(\eta, Y)$ with $\eta$ 
a smooth 1-form, which is close to $(\omega, X)$ but is not topologically equivalent to $(\omega, X)$, that is, $(\omega, X)$ is not stable.

The case when $\omega$ is regular follows from results on bifurcations of vector fields (see $\S 2$ ). The results in this paper are given in $\S 3$ and some preliminaries are given in $\S 2$. We include an appendix containing one of Lara's results and a summary of its proof.

We observe that tangent pairs are also studied by Mol $[\mathbf{6}]$, where he considers a codimension- 1 foliation $\mathcal{G}$ in $\mathbb{P}_{\mathbb{C}}^{3}$ which is invariant by a dimension-1 foliation $\mathcal{F}$ in $\mathbb{P}_{\mathbb{C}}^{3}$. Therefore, locally we have a tangent pair $(\omega, X)$ in $\mathbb{C}^{3}$. Mol proved that there is an open and dense set of dimension- 1 foliations of degree $d$, such that if $\mathcal{F}$ is an element of this subset, then there is no codimension- 1 foliation $\mathcal{G}$ in $\mathbb{P}_{\mathbb{C}}^{3}$ which is invariant by $\mathcal{F}$.

\section{Preliminaries}

Let $U$ be an open set in $\mathbb{R}^{n}$. We denote by $\Lambda^{r}(U)$ (respectively, $\Lambda^{a}(U)$ ) the set of integrable (respectively, analytic) differential 1-forms of class $C^{r}$ in $U, 0 \leqslant r \leqslant \infty$, and endow this set with the Whitney $C^{k}$-topology $(0 \leqslant k \leqslant r)$. So $\omega \wedge \mathrm{d} \omega \equiv 0$ for all $\omega$ in $\Lambda^{r}(U)$ or $\Lambda^{a}(U)$.

A point $p \in U$ is a singularity of $\omega$ if $\omega(p)=0$. We shall assume that the singularities of $\omega$ are isolated. A point $p$ is a non-degenerate singularity of $\omega$ if the linear part of $\omega$ at $p$ is not degenerate, i.e. the determinant of the matrix of the 1-jet of $\omega$ at $p$ is not zero. (See [12] for a classification of singularities of 1-forms.) As $\omega$ is integrable, it determines a codimension- 1 foliation in $U-\{p\}$. This foliation is denoted by $\mathcal{F}_{\omega}$. The foliation $\mathcal{F}_{g \omega}=\mathcal{F}_{\omega}$, for any function $g$ not vanishing in $U$ (and of the same class as $\omega$ ). As we are only interested in the foliation $\mathcal{F}_{\omega}$, we shall identify $g \omega$ with $\omega$, i.e. we consider the Pfaff equation $\omega=0$.

We denote by $\mathfrak{X}^{r}(U)$ (respectively, $\mathfrak{X}^{a}(U)$ ) the set of vector fields of class $C^{r}$ in $U$, $0 \leqslant r \leqslant \infty$ (respectively, analytic), and endow this set with the Whitney $C^{k}$-topology $(0 \leqslant k \leqslant r)$. Let $X \in \mathfrak{X}^{r}(U)$ (respectively, $\left.\mathfrak{X}^{a}(U)\right)$ and $\omega \in \Lambda^{r}(U)$ (respectively, $\omega \in$ $\left.\Lambda^{a}(U)\right)$. We say that $X$ is tangent to $\omega$ if $\omega(x)(X(x))=0$ for all $x$ in $U$. That is, $X(x)$ is tangent to the leaf of $\mathcal{F}_{\omega}$ at $x$. We denote by $\mathfrak{X}^{r}(\omega)$ (respectively, $\mathfrak{X}^{a}(\omega)$ ) the subset of $\mathfrak{X}^{r}(U)$ (respectively, $\mathfrak{X}^{a}(U)$ ) of vector fields tangent to $\omega$. This subset is given the induced topology of $\mathfrak{X}^{r}(U)$ (respectively, $\mathfrak{X}^{a}(U)$ ). We observe that $\mathfrak{X}^{r}(\omega)$ is not empty. Indeed, given a non-identically zero 1 -form $\omega=\sum_{i=1}^{n} a_{i} \mathrm{~d} x_{i}$, then

$$
a_{j} \frac{\partial}{\partial x_{i}}-a_{i} \frac{\partial}{\partial x_{j}} \in \mathfrak{X}^{r}(\omega) \text { for } i \neq j .
$$

In the rest of the paper the notation $(\omega, X)$ is reserved for pairs where $X$ is tangent to $\omega$, which we shall call tangent pairs.

Our interest is in the local behaviour of the pairs $(\omega, X)$ at a given point $p \in U$, which we normally choose to be the origin. So we consider germs at $p$ of integrable differential 1-forms $\omega$ (respectively, vector fields), i.e. equivalence classes of differential 1-forms (respectively, vector fields) under the equivalence of being identical in some neighbourhood of $p$. As usual, properties on germs are checked on a representative of the germ, and throughout the paper we use the same notation for a germ and its representative. 
We denote by $I^{r}(p)$ (respectively, $I^{a}(p)$ ) the set of germs, at $p \in \mathbb{R}^{n}$, of integrable 1-forms of class $C^{r}, 0 \leqslant r \leqslant \infty$ (respectively, analytic), and endow this set with the Whitney $C^{k}$-topology $(0 \leqslant k \leqslant r)$. When $p$ is the origin, the sets $I^{r}(0)$ and $I^{a}(0)$ are denoted by $I^{r}$ and $I^{a}$, respectively. We shall also drop $p$ from the notation below for vector fields when it indicates the origin.

We denote by $V^{r}(p)$ (respectively, $V^{a}(p)$ ) the set of germs at $p \in \mathbb{R}^{n}$ of vector fields of class $C^{r}, 0 \leqslant r \leqslant \infty$ (respectively, analytic), and endow this set with the Whitney $C^{k}$-topology $(0 \leqslant k \leqslant r)$. The subset $V^{r}(\omega)(p)$ (respectively, $\left.V^{a}(\omega)(p)\right)$ of $V^{r}(p)$ (respectively, $\left.V^{a}(p)\right)$ denotes the set of germs of vector fields tangent to $\omega$. This subset is given the induced topology of $V^{r}(p)$ (respectively, $V^{a}(p)$ ).

We need the following definitions (also valid in the analytic category).

\section{Definition 2.1.}

(i) Two germs $\omega \in I^{r}$ and $\eta \in I^{r}(p)$ are topologically equivalent if there exists a germ of homeomorphism $h: \mathbb{R}^{n}, 0 \rightarrow \mathbb{R}^{n}, p$ such that $h$ is a topological equivalence between the (germs of) foliations $\mathcal{F}_{\omega}$ and $\mathcal{F}_{\eta}$, i.e. taking representatives, the image under $h$ of the leaves of $\mathcal{F}_{\omega}$ are the leaves of $\mathcal{F}_{\eta}$.

(ii) A germ $\omega \in I^{r}$ is $C^{s}$-topologically stable if a representative $\omega \in \Lambda^{r}(U)$ in some neighbourhood $U$ of the origin satisfies the following. For any neighbourhood $U^{\prime} \subset$ $U$ of the origin, there exists a neighbourhood $\mathcal{U} \subset \Lambda^{r}(U)$ of $\omega$ in the Whitney $C^{s_{-}}$ topology such that any $\eta \in \mathcal{U}$ has a germ at some point $p \in U^{\prime}$ which is topologically equivalent to the germ of $\omega$ at the origin.

The notions of topological equivalence of germs and stable germs of vector fields are defined in similar ways for differential 1-forms (Definition 2.1), where now the homeomorphism $h$ preserves trajectories, i.e. oriented orbits of the vector field.

Definition 2.2. Let $(\omega, X) \in I^{r} \times V^{r}$ and $(\eta, Y) \in I^{r}(p) \times V^{r}(p)$ be two tangent pairs. We say that $(\omega, X)$ and $(\eta, Y)$ are topologically equivalent if there exists a germ of homeomorphism $h: \mathbb{R}^{n}, 0 \rightarrow \mathbb{R}^{n}, p$ such that

(i) $h$ is a topological equivalence between $\omega$ and $\eta$,

(ii) if $F$ is a leaf of $\mathcal{F}_{\omega}$, then $\left.h\right|_{F}$ is a topological equivalence between $\left.X\right|_{F}$ and $\left.Y\right|_{h(F)}$.

We are seeking tangent pairs that are topologically stable, that is, tangent pairs with the property that they are topologically equivalent to any nearby tangent pair. Here the term 'nearby' needs to be clarified. The set $\Lambda^{r}(U)$ (and $I^{r}$ ) can be given the Whitney $C^{s}$-topology $(s \leqslant r)$ and $\mathfrak{X}^{r}(U)$ (and $\left.V^{r}\right)$ the Whitney $C^{t}$-topology $(t \leqslant r)$, so the set of tangent pairs is given the induced topology of the product $C^{s} \times C^{t}$-topology. It is also of interest to study germs of vector fields that are tangent to a fixed foliation $\mathcal{F}_{\omega}$, that is, vector fields in $\mathfrak{X}^{r}(\omega)$ (or in $V^{r}(\omega)$ ). 
Definition 2.3. Let $\omega \in I^{r}$ and $X \in V^{r}(\omega)$.

(i) We say that $X$ is $C^{t}$-topologically stable if there exists a neighbourhood $\mathcal{V} \subset V^{r}(\omega)$ of $X$ in the Whitney $C^{t}$-topology such that, for any $Y \in \mathcal{V}$, the pair $(\omega, X)$ is topologically equivalent to $(\omega, Y)$.

(ii) We say that the pair $(\omega, X)$ is $C^{s, t}$-topologically stable if a representative $(\omega, X) \in$ $\Lambda^{r}(U) \times \mathfrak{X}^{r}(U)$ in some neighbourhood $U$ of the origin satisfies the following. For any neighbourhood $U^{\prime} \subset U$ of the origin, there exist neighbourhoods $\mathcal{U} \subset \Lambda^{r}(U)$ of $\omega$ in the Whitney $C^{s}$-topology and $\mathcal{V} \subset \mathfrak{X}^{r}(U)$ of $X$ in the Whitney $C^{t}$-topology, such that any pair $(\eta, Y) \in \mathcal{U} \times \mathcal{V}$ has a germ at some point $p \in U^{\prime}$ which is topologically equivalent to the germ of $(\omega, X)$ at the origin.

Our investigation is motivated by the following question: given a topologically stable pair $(\omega, X)$ (in some topology), are $\omega$ and/or $X$ topologically stable?

When $n=2$ the integral curves of $X \in V^{r}(\omega)$ coincide with the leaves of $\mathcal{F}_{\omega}$. Therefore, $(\omega, X)$ is stable if and only if $X$ is topologically stable (see, for example, [2] for a classification of stable germs of vector fields in $\left.\mathbb{R}^{2}, 0\right)$. However, the problem is of a different nature when $n \geqslant 3$.

If $\omega(0) \neq 0$, the problem becomes that of the classification of families of vector fields. In fact we can fix the 1-form $\omega$ (for example, $\left.\omega=\mathrm{d} x_{n}\right)$ and a pair $(\omega, X) \in I^{r} \times V^{r}$ is stable if and only if $X$ is stable in $V^{r}(\omega)$ (see Remark 3.3(1)). For example, when $n=3$ and $\omega=\mathrm{d} x_{3}$ we have the following generic types which are topologically stable in $V^{r}\left(\mathrm{~d} x_{3}\right)$ in the topologies $C^{0}, C^{1}, C^{2}$ and $C^{3}$, respectively (see $[\mathbf{9}]$ ):

(i) $X(0) \neq 0$;

(ii) $X_{\mid x_{3}=c}$ has a hyperbolic singularity at $(0,0, c)$;

(iii) $X$ has a saddle-node singularity at the origin;

(iv) birth of a limit cycle.

In case (ii), for example, the pair $(\omega, X)$ is $C^{1,1}$-stable in $I^{1} \times V^{1}$ but $X$ is clearly not stable in the set of germs of vector fields in $\mathbb{R}^{3}, 0$. Therefore, the stability of the pair $(\omega, X)$ does not imply the stability of $X$ in the set of germs of vector fields. However, it is clear from Definition 2.3 that if $(\omega, X)$ is $C^{s, t}$-topologically stable in the set of tangent pairs in $\Lambda^{r} \times \mathfrak{X}^{r}$, then $X$ is $C^{t}$-topologically stable in $V^{r}(\omega)$.

We investigate next the stability of $\omega$ when $(\omega, X)$ is stable, and assume that $\omega(0)=0$. Suppose that $(\omega, X)$ is stable (in some topology). Given any 1-form $\eta$ close to $\omega$, in order to deduce that $\eta$ is equivalent to $\omega$, we need to find a vector field $Y$ close to $X$ and tangential to $\eta$. Then the pair $(\eta, Y)$ is equivalent to $(\omega, X)$, as this pair is stable, and hence $\eta$ is equivalent to $\omega$. The main difficulty is in finding such $Y$ tangent to $\eta$. In the next section, we characterize all analytic vector fields tangent to an analytic 1-form $\omega$ in terms of $\omega$. This allows us to deduce the stability of $\omega$ from that of $(\omega, X)$. 


\section{Topological stability of pairs $(\omega, X)$}

We consider germs of analytic pairs $(\omega, X)$ and write $\omega=\sum_{i=1}^{n} a_{i} \mathrm{~d} x_{i}$, where the $a_{i}$ are germs of analytic functions. We set $a=\left(a_{1}, \ldots, a_{n}\right)$ and denote by $\tilde{a}: \mathbb{C}^{n}, 0 \rightarrow \mathbb{C}^{n}, 0$ the germ of the complexification of $a$.

Theorem 3.1. Let $\omega \in I^{a}$ and suppose that the complexified foliation has an isolated singularity at the origin. Let $(\omega, X) \in I^{a} \times V^{a}$ be a germ of a $C^{1,1}$-topologically stable analytic tangent pair. Then $\omega$ is $C^{1}$-topologically stable in $I^{a}$.

Proof. We start by finding the germs of analytic vector fields tangent to $\omega$. If $X=$ $\left(X_{1}, \ldots, X_{n}\right)$, then it is tangent to $\omega$ if and only if

$$
a_{1} X_{1}+\cdots+a_{n} X_{n}=0
$$

Denote by $\mathcal{O}_{n}$ the set of germs of holomorphic functions $\mathbb{C}^{n}, 0 \rightarrow \mathbb{C}$. Let $G:\left(\mathcal{O}_{n}\right)^{n} \rightarrow$ $\mathcal{O}_{n}$ be the homomorphism defined by $G\left(g_{1}, \ldots, g_{n}\right)=\sum_{i=1}^{n} g_{i} \tilde{a}_{i}$. As the map $\tilde{a}$ is finite, it follows that the kernel of $G$ is generated by $\tilde{a}_{i} e_{j}-\tilde{a}_{j} e_{i}, i<j$, where the vector $e_{i}$ has 1 in the $i$ th place and zeros elsewhere. (This follows from the fact that $\tilde{a}_{1}, \ldots, \tilde{a}_{n}$ form an $\mathcal{O}_{n}$-regular sequence (see $\left[\mathbf{5}\right.$, p. 123] for definition). Then $\tilde{a}_{n}$ is $\mathcal{O}_{n} / \mathcal{O}_{n}\left(\tilde{a}_{1}, \ldots, \tilde{a}_{n-1}\right)$ regular. Suppose that $g=\left(g_{1}, \ldots, g_{n}\right)$ lies in the kernel of $G$. We have $\tilde{a}_{n} g_{n}=0$ in $\mathcal{O}_{n} / \mathcal{O}_{n}\left(\tilde{a}_{1}, \ldots, \tilde{a}_{n-1}\right)$, and so $g_{n}=0$ in the quotient ring. Writing $g_{n}=\alpha_{1} \tilde{a}_{1}+\cdots+$ $\alpha_{n-1} \tilde{a}_{n-1}$, we subtract $\sum_{i=1}^{n-1} \alpha_{i}\left(\tilde{a}_{i} e_{n}-\tilde{a}_{n} e_{i}\right)$ from $g$ to obtain an element of the kernel whose last component vanishes. The result then follows by induction.)

The real parts of the generators of the kernel of $G$ generate the real part of that kernel. As the $a_{i}$ are real, it follows that any $X$ satisfying equation (3.1) can be written in the form

$$
\sum_{i, j=1, i<j}^{n} \alpha_{i j}\left(a_{i} e_{j}-a_{j} e_{i}\right),
$$

where $\alpha_{i j}$ are germs of real analytic functions.

Suppose now that $\omega$ is not $C^{1}$-topologically stable and let $(\omega, X)$ be a $C^{1,1}$-stable tangent pair, with $X$ as in (3.2). We take representatives of $\omega$ and $X$ in some neighbourhood $U$ of the origin. (We can still write $X$ as in (3.2), with its coefficients being the representatives of the germs $\alpha_{i j}$ in $U$, shrinking $U$ if necessary.)

Let $\eta=\sum_{i=1}^{n} b_{i} \mathrm{~d} x_{i}$ be an analytic 1 -form $C^{1}$-close to $\omega$ in $\Lambda^{a}(U)$ but not topologically equivalent to $\omega$. The vector field $Y=\sum_{i, j=1, i<j}^{n} \alpha_{i j}\left(b_{i} e_{j}-b_{j} e_{i}\right)$ in $\mathfrak{X}^{a}(U)$, with $\alpha_{i j}$ the coefficients of $X$ in $U$, is tangent to $\eta$. It is clear that $Y$ is $C^{1}$-close to $X$ and the pair $(\omega, X)$ is not topologically equivalent to $(\eta, Y)$, which contradicts the hypothesis. Therefore, $\omega$ is $C^{1}$-topologically stable.

We seek now examples of stable tangent pairs with $\omega(0)=0$. We shall suppose that the origin of $\mathbb{R}^{n}$ is a non-degenerate singularity of $\omega$ in $I^{\infty}$ or in $I^{a}$. Then we can write its 1 -jet at the origin, in a suitable coordinate system, in the form $j^{1} \omega=\mathrm{d}\left(x_{1}^{2}+\cdots+\right.$ $x_{k}^{2}-x_{k+1}^{2}-\cdots-x_{n}^{2}$ ) (see, for example, $[\mathbf{1}]$ ). We say that the singularity at the origin is of index $k$. When $n=3$ we have the following result. 


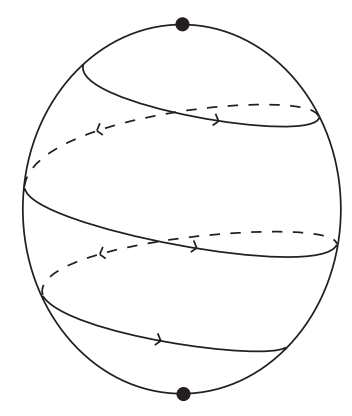

Figure 1. Morse-Smale vector field of north-pole-south-pole type.

(a)

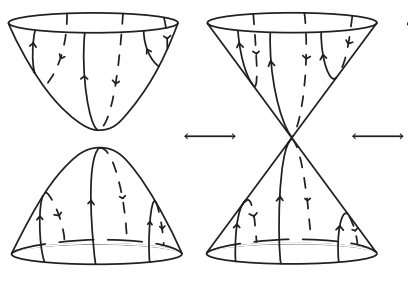

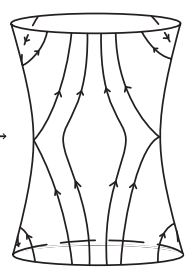

(b)

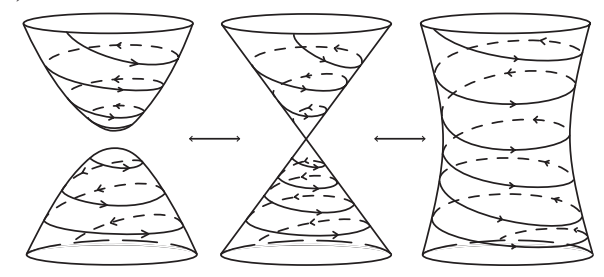

Figure 2. Generic vector fields tangent to the foliation of $\mathrm{d} f=\mathrm{d}\left(x_{1}^{2}+x_{2}^{2}-x_{3}^{2}\right)$.

\section{Theorem 3.2.}

(a) Let $\omega \in I^{\infty}$ and suppose that $\omega$ has a non-degenerate singularity of index 3 (or 0 ) at the origin in $\mathbb{R}^{3}$.

(a1) There exists an open and dense set $\mathcal{A}^{\prime} \subset V^{2}(\omega)$ of vector fields $C^{2}$-stable in $V^{2}(\omega)$ whose restrictions to each leaf of $\mathcal{F}_{\omega}$ are of Morse-Smale north-polesouth-pole type (see Figure 1).

(a2) For any $X \in \mathcal{A}^{\prime}$, the pair $(\omega, X)$ is $C^{3,2}$-topologically stable in $I^{\infty} \times V^{2}$.

(b) Let $\omega \in I^{a}$ and suppose that $\omega$ has a non-degenerate singularity of index 2 (or 1) at the origin in $\mathbb{R}^{3}$.

(b1) There exist open sets $\mathcal{B}^{\prime}, \mathcal{C}^{\prime} \subset V^{2}(\omega)$ with $\mathcal{B}^{\prime} \cup \mathcal{C}^{\prime}$ dense in $V^{2}(\omega)$ such that

(i) the vector fields in $\mathcal{B}^{\prime}$ are $C^{1}$-stable in $V^{1}(\omega)$ and topologically equivalent to $\left(x_{2}+x_{3},-x_{1}-x_{3}, x_{1}-x_{2}\right)$ (see Figure $2 a$ ),

(ii) the vector fields in $\mathcal{C}^{\prime}$ are $C^{2}$-stable in $V^{2}(\omega)$ and topologically equivalent to $\left(-x_{2}-x_{1} x_{3}, x_{1}-x_{2} x_{3},-\left(x_{1}^{2}+x_{2}^{2}\right)\right.$ ) (see Figure $2 b$ ).

(b2) For any $X \in \mathcal{B}^{\prime}$, the pair $(\omega, X)$ is $C^{2,1}$-topologically stable in $I^{a} \times V^{1}$.

(b3) For any $X \in \mathcal{C}^{\prime}$, the pair $(\omega, X)$ is $C^{3,2}$-topologically stable in $I^{a} \times V^{2}$.

Proof. (a1) We can assume, without loss of generality, that $j^{1} \omega=\mathrm{d}\left(x_{1}^{2}+x_{2}^{2}+x_{3}^{2}\right)$. Then $\omega$ has an integrating factor [7]; that is, there exist germs of functions $f, g: \mathbb{R}^{3}, 0 \rightarrow$ $\mathbb{R}$, with 0 a non-degenerate critical point of $f$ and $g(0) \neq 0$, such that $\omega=g \mathrm{~d} f$. A $C^{\infty}$ 
change of coordinates sets $\mathrm{d} f=\mathrm{d}\left(x_{1}^{2}+x_{2}^{2}+x_{3}^{2}\right)$. As $\omega$ is a multiple of $\mathrm{d} f$, the topological stability of $X \in V^{2}(\omega)$ is the same as that of $(f, X)$ in Lara's work. The result then follows by Lara's theorem (Theorem A $2(\mathrm{a})$ ). If $\phi$ is the diffeomorphism that sets $\omega$ to $g \mathrm{~d} f$, then we take $\mathcal{A}^{\prime}=\left\{\phi_{*}^{-1} Z, Z \in \mathcal{A}\right\}$, where $\mathcal{A}$ is as in Appendix A.

(a2) Let $(\omega, X)$ be a tangent pair and assume, without loss of generality, that $\omega=$ $\mathrm{d}\left(x_{1}^{2}+x_{2}^{2}+x_{3}^{2}\right)$. Let $(\eta, Y)$ be a tangent pair close to $(\omega, X)$ in some topology to be determined below. Then there exists a $C^{\infty}$-diffeomorphism $h$ such that $\left(h_{*} \eta, h_{*} Y\right)=$ $\left(w, h_{*} Y\right)$. In order to use (a1), $X$ must be in $\mathcal{A}$ and $h_{*} Y$ must satisfy the properties given below. We claim that such a diffeomorphism exists as long as $\eta$ is $C^{3}$-close to $\omega$. We prove the claim in two steps. We first show that $h$ can be taken to have a 3 -jet, at the origin, the identity. We then show that, for such an $h, h_{*} Y$ satisfies the desired properties.

We set $\eta=\mathrm{d} f$ (see the argument in (a1)) and make linear changes of coordinates (close to the identity) so that $j^{2} f=x_{1}^{2}+x_{2}^{2}+x_{3}^{2}$. Write $f=f_{2}+f_{3}+f_{4}+\cdots$, where $f_{i}$ are homogeneous polynomials of degree $i$. As $\eta$ is $C^{3}$-close to $\omega=\mathrm{d}\left(x_{1}^{2}+x_{2}^{2}+x_{3}^{2}\right)$, the functions $f_{3}$ and $f_{4}$ are $C^{3}$ - and $C^{4}$-close to zero, respectively, and indeed $C^{\infty}$-close to zero. Let $h=\left(x_{1}+k_{2}, x_{2}+l_{2}, x_{3}+m_{2}\right)$, where $k_{2}, l_{2}, m_{2}$ are homogeneous polynomials of degree 2 . Then the homogeneous part of degree 3 in $f \circ h$ is given by

$$
2 x_{1} k_{2}+2 x_{2} l_{2}+2 x_{3} m_{2}+f_{3} .
$$

As $f_{3}$ is $C^{\infty}$-close to zero, we can make the above expression vanish with $k_{2}, l_{2}, m_{2}$ well chosen and $C^{\infty}$-close to zero. For example, we can take

$$
\begin{aligned}
k_{2} & =-\frac{f_{3}\left(x_{1}, x_{2}, x_{3}\right)-f_{3}\left(0, x_{2}, x_{3}\right)}{2 x_{1}}, \\
l_{2} & =-\frac{f_{3}\left(0, x_{2}, x_{3}\right)-f_{3}\left(0,0, x_{3}\right)}{2 x_{2}}, \\
m_{2} & =-\frac{f_{3}\left(0,0, x_{3}\right)}{2 x_{3}} .
\end{aligned}
$$

We can now suppose that $j^{3} f=x_{1}^{2}+x_{2}^{2}+x_{3}^{2}$. Repeating the above process, we eliminate the terms of degree 4 in $f$ by a diffeomorphism of the form $h=\left(x_{1}+k_{3}, x_{2}+l_{3}, x_{3}+m_{3}\right)$, where $k_{3}, l_{3}, m_{3}$ are homogeneous polynomials of degree 3 satisfying

$$
2 x_{1} k_{3}+2 x_{2} l_{3}+2 x_{3} m_{3}+f_{4}=0 .
$$

We choose, as above, a solution with $k_{3}, l_{3}$ and $m_{3} C^{\infty}$-close to zero. We can now suppose that $j^{4} f=x_{1}^{2}+x_{2}^{2}+x_{3}^{2}$. One can show, by applying [11, Proposition 4.4.1], that there exists a germ of a diffeomorphism $h$, with $j^{3} h$ the identity, such that $f \circ h=x_{1}^{2}+x_{2}^{2}+x_{3}^{2}$.

(The idea in the following step was suggested by the referee.) We know that $h_{*} Y \in$ $V^{2}(\omega)$ as $h_{*} \eta=\omega$. Let $\lambda$ and $b$ (respectively, $\lambda^{\prime}$ and $b^{\prime}$ ) be the constants associated to $X$ (respectively, $h_{*} Y$ ) in Appendix $\mathrm{A}$, and suppose that $X \in \mathcal{A}$. It follows from the proof of Theorem A 2 that, for $\left(\omega, h_{*} Y\right)$ to be topologically equivalent to $(\omega, X)$, it is sufficient for $h_{*} Y \in \mathcal{A}$ (i.e. $\lambda^{\prime} b^{\prime} \neq 0$ ) and for $b^{\prime}$ to have the same sign as $b$. The constants $\lambda^{\prime}$ and $b^{\prime}$ 
depend only on $j^{2}\left(h_{*} Y\right)$ at the origin. However, the 3 -jet of $h$ is the identity, so $\lambda^{\prime}$ and $b^{\prime}$ depend only on $j^{2} Y$ at the origin. If we take $Y$ to be $C^{2}$-close to $X$, then $\left(\lambda^{\prime}, b^{\prime}\right)$ is close to $(\lambda, b)$ (see the proof of Theorem A 2). So, in this case, $h_{*} Y \in \mathcal{A}$ and $b^{\prime}$ have the same sign as $b$. Consequently, $\left(\omega, h_{*} Y\right)$ is topologically equivalent to $(\omega, X)$.

(b) The difference here is that if $\omega$ has index 2 (or 1 ) it has no integrating factor in the smooth category but does have one in the analytic category [8]. So, assuming that $\omega$ is analytic, we can proceed as in (a). Then the results in (b1) follow from Lara's work (as in the proof of (a1)) and those in (b2) and (b3) by using the same argument as in the proof of (a2). If $\phi$ is the diffeomorphism that sets $\omega$ to $g \mathrm{~d} f$, then we take $\mathcal{B}^{\prime}=\left\{\phi_{*}^{-1} Z, Z \in \mathcal{B}\right\}$ and $\mathcal{C}^{\prime}=\left\{\phi_{*}^{-1} Z, Z \in \mathcal{C}\right\}$, where $\mathcal{B}$ and $\mathcal{C}$ are as in Appendix A.

\section{Remark 3.3.}

(1) Following the argument in the proof of Theorem 3.2 (a2), the study of the topological stability of tangent pairs $(\omega, X)$ when $\omega$ is regular also reduces to the study of $X \in V^{r}(\omega)$. Then the pairs $(w, X)$ in $\mathbb{R}^{3}, 0$ in $\S 2$ are topologically stable in the topologies $C^{0,0}, C^{1,1}, C^{2,2}$ and $C^{3,3}$, respectively, for the cases (i)-(iv).

(2) Lara's results for $n \geqslant 4$ (Remark A 3) can also be generalized to $C^{\infty}$ (respectively, analytic) 1 -forms $\omega$ if the index is distinct from 2 or $n-2$ (respectively, equal to 2 or $n-2)$. The topological stability of pairs $(\omega, X)$, in appropriate topologies, can be dealt with as in Theorem 3.2.

In Theorem 3.2(b) when the index of the singularity of $\omega$ is 2 or 1 , we restricted ourselves to the set analytic 1-forms. We show below that there are no topologically stable pairs $(\omega, X)$ in the smooth category when $\omega$ is analytic and has a non-degenerate singularity of index 2 or $n-2$.

Medeiros showed in $[\mathbf{3}]$ that the stable 1-form $\omega^{1}=x \mathrm{~d} x+y \mathrm{~d} y-\left(z_{1} \mathrm{~d} z_{1}+\cdots+z_{l} \mathrm{~d} z_{l}\right)$, $l=n-2$ (the case of Theorem 3.2 (b) when $n=3$ ), in the set of analytic 1-forms is not topologically stable in the set of smooth 1-forms. Indeed, consider the following 1-form:

$$
\beta= \begin{cases}\frac{\varepsilon r \exp (-1 / r)}{x^{2}+y^{2}}(-y \mathrm{~d} x+x \mathrm{~d} y) & \text { if } r>0, \\ 0 & \text { if } r \leqslant 0,\end{cases}
$$

where $r=x^{2}+y^{2}-\left(z_{1}^{2}+\cdots+z_{l}^{2}\right), l=n-2$. One can choose $\varepsilon$ small enough so that $\beta$ is $C^{\infty}$-close to zero. The 1 -form $\omega^{1}+\beta$ is integrable, is $C^{\infty}$-close to $\omega^{1}$ but is not topologically equivalent to $\omega^{1}$, as the two 1 -forms have distinct limit sets $[\mathbf{3}]$. We use this result to show the following.

Theorem 3.4. Let $\omega \in I^{a}$. Suppose that the origin is a non-degenerate singularity of index 2 or $n-2$ of $\omega$. Then, for any $X \in V^{\infty}(\omega)$, there exists a tangent pair $(\eta, Y) \in$ $I^{\infty} \times V^{\infty}$ which is $C^{\infty, 1}$-close to $(\omega, X)$ but is not topologically equivalent to $(\omega, X)$.

Proof. As $\omega$ is analytic, it admits an analytic first integral [8]. In fact, $\omega$ is analytically equivalent to $\omega^{1}=x \mathrm{~d} x+y \mathrm{~d} y-\left(z_{1} \mathrm{~d} z_{1}+\cdots+z_{l} \mathrm{~d} z_{l}\right)$, where $l=n-2$, in some coordinate system. So it is enough to consider tangent pairs $\left(\omega^{1}, X\right)$. 
Given a tangent pair $\left(\omega^{1}, X\right),\left(\omega^{1}, j^{k} X\right)$ is also a tangent pair, for any $k$-jet of $X$ at the origin, with $k \geqslant 1$ [4, Lemma II-3]. Observe that $j^{k} X$ is $C^{k}$-close to $X$. Now, as the tangent pair $\left(\omega^{1}, j^{k} X\right)$ is analytic and the complexified foliation of $\omega^{1}$ has an isolated singularity, we have

$$
j^{k} X=\sum_{i, j=1, i<j}^{n} \alpha_{i j}\left(a_{i} e_{j}-a_{j} e_{i}\right)
$$

for some germs of analytic function $\alpha_{i j}$ (proof of Theorem 3.1).

Let $\beta$ be as in (3.3) and set $\tilde{\omega}=\omega^{1}+\beta$. We seek a vector field $Z \in V^{\infty}\left(C^{k}\right.$-close to zero), such that $j^{k} X+Z$ is tangent to $\tilde{\omega}$. So we need $\tilde{\omega}\left(j^{k} X+Z\right)=0$, that is $\left(\omega^{1}+\beta\right)\left(j^{k} X+Z\right)=0$. Equivalently,

$$
\omega^{1}(Z)+\beta\left(j^{k} X+Z\right)=0 .
$$

Let $Z$ be identically zero when $r=x^{2}+y^{2}-\left(z_{1}^{2}+\cdots+z_{l}^{2}\right) \leqslant 0$, and write $Z=$ $\left(Z_{1}, \ldots, Z_{n}\right)$ when $r>0$. Substituting in (3.4) yields an equation that can be rearranged as follows:

$$
x\left(Z_{1}+\delta Z_{2}+\alpha_{12} x \delta\right)+y\left(-\delta Z_{1}+Z_{2}+\alpha_{12} y \delta\right)+\sum_{j=3}^{n} z_{j-2}\left(-Z_{j}+\alpha_{2 j} x \delta-\alpha_{1 j} y \delta\right)=0,
$$

where $\delta=\varepsilon r \exp (-1 / r) /\left(x^{2}+y^{2}\right)$ and $\alpha_{i j}$ are the coefficients of $j^{k} X$ above. We can now find $Z$ by setting the quantities in brackets to be zero. We obtain

$$
\begin{aligned}
& Z_{1}=\alpha_{12}(-x+y \delta) \delta /\left(1+\delta^{2}\right), \\
& Z_{2}=-\alpha_{12}(y+x \delta) \delta /\left(1+\delta^{2}\right), \\
& Z_{j}=\left(\alpha_{2 j} x-\alpha_{1 j} y\right) \delta, \quad j=3, \ldots, n .
\end{aligned}
$$

The vector field $j^{k} X+Z$ is tangent to $\tilde{\omega}$ and is $C^{k}$-close to $X$. As $\tilde{\omega}$ is not equivalent to $\omega^{1}[\mathbf{3}]$, it follows that $\left(\omega^{1}, X\right)$ is not topologically equivalent to $\left(\tilde{\omega}, j^{k} X+Z\right)$, that is, $\left(\omega^{1}, X\right)$ is not $C^{\infty, k}$-topologically stable. Clearly, we can take $k=1$.

Acknowledgements. We are very much indebted to the referee for very useful comments and for the argument in the proof of Theorem 3.2. We also thank J. L. Arraut and J. W. Bruce for useful discussions. The work of L.F.M. was supported by FAPESP of Brazil, Grant no. Proc 05/55900-0.

\section{Appendix A.}

We state in this appendix one of Lara's results in [4] and summarize his proof. Before doing so, we recall a classification theorem of Takens. Let $X \in V^{k}$ and denote by $X_{1}$ the 1-jet of $X$ at the origin. Denote by $H^{m}$ the real vector space of vector fields whose coefficients are homogeneous polynomials of degree $m$. Let $T^{m}: H^{m} \rightarrow H^{m}$ be the linear map given by $T^{m}(Z)=\left[X_{1}, Z\right]$, where $[\cdot, \cdot]$ denotes the Lie bracket. One can write $H^{m}=B^{m} \oplus G^{m}$, where $B^{m}=\operatorname{Im}\left(T^{m}\right)$ and $G^{m}$ is a complementary space. 
Theorem A 1 (Takens [10, Theorem 2.1]). Let $X, X_{1}, B^{m}$ and $G^{m}$ be as above. Then, for $l \leqslant k$, there is a germ of a $C^{\infty}$-diffeomorphism $\phi: \mathbb{R}^{n}, 0 \rightarrow \mathbb{R}^{n}, 0$ such that $\phi_{*}(X)=X^{\prime}$ is of the form

$$
X^{\prime}=X_{1}+g_{2}+\cdots+g_{l}+R_{l},
$$

where $g_{i} \in G^{i}, i=2, \ldots, l$, and $R_{l}$ is a vector field, the component functions of which all have zero l-jet.

The proof is by induction on $l$. For $l=1$, the assertion is trivially true. Suppose that $X=X_{1}+g_{2}+\cdots+g_{l-1}+R_{l-1}$. We can then write $R_{l-1}=b_{l}+g_{l}+R_{l}$ with $b_{l} \in B^{l}$. There exists $Y \in H^{l}$ such that $b_{l}=T^{l}(Y)$. The diffeomorphism $\phi$ is then given by integrating $Y$ over time $t=-1$.

Lara studied in [4] the local topological stability of pairs $(\mathrm{d} f, X)$, where $f$ is a function with a Morse singularity. A complete classification of stable pairs is given in [4] for the cases when $n=3,4$, and for $n \geqslant 5$ if the index of the singularity of $f$ is 0 or $n$. One can take, without loss of generality, the function $f$ in a normal form. The main theorem in [4] for the case $n=3$ is the following.

Theorem A 2 (Lara [4, Theorem 1]).

(a) If $f=x_{1}^{2}+x_{2}^{2}+x_{3}^{2}$, then there exists an open and dense set $\mathcal{A} \subset V^{2}(\mathrm{~d} f)$ of vector fields $C^{2}$-stable in $V^{2}(\mathrm{~d} f)$ whose restrictions to each leaf of $\mathcal{F}_{\mathrm{d} f}$ are of Morse-Smale north-pole-south-pole type (see Figure 1).

(b) If $f=x_{1}^{2}+x_{2}^{2}-x_{3}^{2}$, then there exist open sets $\mathcal{B}, \mathcal{C} \subset V^{2}(\mathrm{~d} f)$ with $\mathcal{B} \cup \mathcal{C}$ dense in $V^{2}(\mathrm{~d} f)$ such that

(i) the vector fields in $\mathcal{B}$ are $C^{1}$-stable in $V^{1}(\mathrm{~d} f)$ and topologically equivalent to $\left(x_{2}+x_{3},-x_{1}-x_{3}, x_{1}-x_{2}\right)$ (see Figure $2 a$ ),

(ii) the vector fields in $\mathcal{C}$ are $C^{2}$-stable in $V^{2}(\mathrm{~d} f)$ and topologically equivalent to $\left(-x_{2}-x_{1} x_{3}, x_{1}-x_{2} x_{3},-\left(x_{1}^{2}+x_{2}^{2}\right)\right)$ (see Figure $2 b$ ).

Proof. The proof in [4] is very detailed and lengthy. We give a brief summary here, highlighting the main ideas. The theorem is proved in several steps, with the first step concerning the reduction of $X \in V^{k}(\mathrm{~d} f)$ to some pre-normal form.

(a) A vector field $X$ tangent to the level sets of $f$ has the properties that $X(0)=0$ and $X_{1}=D X(0)$ is given by an antisymmetric matrix. Thus, the eigenvalues of $X_{1}$ are $\lambda_{1}=0, \lambda_{2}=\mathrm{i} \lambda$ and $\lambda_{3}=-\mathrm{i} \lambda(\lambda \in \mathbb{R})$. One can then change coordinates via a rotation and write $X_{1}=\lambda T$ with

$$
T=-x_{2} \frac{\partial}{\partial x_{1}}+x_{1} \frac{\partial}{\partial x_{2}} .
$$

Using Takens's theorem (Theorem A 1), there exists a diffeomorphism $\phi$ such that $\phi_{*} X \in$ $V^{2}(\mathrm{~d} f)$ and $\phi_{*} X=\lambda T+X_{2}+R_{2}$, with $j^{2} R_{2} \equiv 0$ and $X_{2}$ in the kernel of the linear map $T^{2}$. The kernel of $T^{2}$ is generated by $x_{3} T$ and

$$
x_{3} R-r^{2} \frac{\partial}{\partial x_{3}},
$$


with

$$
R=x_{1} \frac{\partial}{\partial x_{1}}+x_{2} \frac{\partial}{\partial x_{2}} \quad \text { and } \quad r^{2}=x_{1}^{2}+x_{2}^{2}
$$

So

$$
X_{2}=a x_{3} T+b\left(x_{3} R-r^{2} \frac{\partial}{\partial x_{3}}\right) \text { for some } a, b \in \mathbb{R} \text {. }
$$

Let

$$
\mathcal{A}=\left\{X \in V^{2}(\mathrm{~d} f): \lambda b \neq 0\right\} .
$$

If $X \in \mathcal{A}$, then the restriction of $\phi_{*} X$ (and hence of $X$ ) to each leaf of $\mathcal{F}_{\mathrm{d} f}$ is of Morse-Smale north-pole-south-pole type, and the poles are the only singularities. The sign of $b$ determines which pole is an attractor or repulsor. Using blow-up, Lara showed that, in some neighbourhood of the origin, the poles of $\phi_{*} X=\lambda T+X_{2}+R_{2}$ on the spheres $S_{r}=f^{-1}(r)$ occur inside some cone $C(s)=\left\{\left(x_{1}, x_{2}, x_{3}\right): x_{1}^{2}+x_{2}^{2}=s x_{3}^{2}\right\}, s>0$.

Let $Y \in \mathcal{A}$, then there exists a diffeomorphism $\psi$ such that $\psi_{*} Y=\lambda^{\prime} T+Y_{2}+R_{2}^{\prime}$ (as above for $X$ ). Let $b^{\prime}$ be the constant associated to $Y_{2}$, and suppose that $b^{\prime}$ has the same sign as $b$. On any given sphere $S_{r}$ (with $r$ small enough), the type of a given pole of $\left.\psi_{*} Y\right|_{S_{r}}$ (attractor/repulsor) is the same as that of the nearby one of $\left.\phi_{*} X\right|_{S_{r}}$. We can suppose that the cone $C(s)$ contains the singularities of $\psi_{*} Y$ (otherwise take $C(s)$ as the cone associated to $\left.\psi_{*} Y\right)$. Lara then constructed the topological conjugation $h$ between $\left(\mathrm{d} f, \phi_{*} X\right)$ and $\left(\mathrm{d} f, \psi_{*} Y\right)$ as follows:

(1) $h(x)=x$ if $x \in \partial C(s)$ with $x_{3}>0$;

(2) $h$ sends the north poles (respectively, south poles) of $\phi_{*} X$ on $S_{r}$ to the north poles (respectively, south poles) of $\psi_{*} Y$ on the same sphere;

(3) if $x$ is not a singularity of $\phi_{*} X$, let $t(x)$ be the time such that $\phi_{*} X_{t(x)}(x) \in \partial C(s)$; then $h(x)=\psi_{*} Y_{-t(x)}\left(\phi_{*} X_{t(x)}(x)\right)$ (observe that one can now conclude that $(\mathrm{d} f, X)$ and $(\mathrm{d} f, Y)$ are topologically equivalent).

Let $Y \in V^{2}(\mathrm{~d} f)$ be $C^{2}$-close to $X \in \mathcal{A}$. Then $Y \in \mathcal{A}$ and $b^{\prime}$ has the same sign as $b$. Indeed, the constant $\lambda^{\prime}$ is obtained from the eigenvalues of $j^{1} Y$ at the origin, so $\lambda^{\prime}$ is close to $\lambda$, and hence $\lambda^{\prime} \neq 0$. Also, $Y_{1}=\lambda^{\prime} T$ and $X_{1}=\lambda T$, so the linear map $T_{Y}^{2}=\left[Y_{1},-\right]: H^{2} \rightarrow H^{2}$ is a scalar multiple of $T_{X}^{2}=\left[X_{1},-\right]: H^{2} \rightarrow H^{2}$. Consider the decomposition $H^{2}=B^{2} \oplus G^{2}$ with $B^{2}=\operatorname{Im}\left(T_{X}^{2}\right)=\operatorname{Im}\left(T_{Y}^{2}\right)$ and $G^{2}=\operatorname{ker}\left(T_{X}^{2}\right)=\operatorname{ker}\left(T_{Y}^{2}\right)$ in the sketch of the proof of Takens's theorem (Theorem A 1). Then the hypothesis implies that $Y_{2}$ is $C^{\infty}$-close to $X_{2}$. Therefore, $b^{\prime}$ is close to $b$, so it has the same sign as $b$. Consequently, $(\mathrm{d} f, Y)$ is equivalent to $(\mathrm{d} f, X)$.

(i) Suppose now that $f=x_{1}^{2}+x_{2}^{2}-x_{3}^{2}$. If $X \in V^{2}(\mathrm{~d} f)$, then

$$
D X(0)=\left(\begin{array}{ccc}
0 & \alpha & \beta \\
-\alpha & 0 & \gamma \\
\beta & \gamma & 0
\end{array}\right)
$$


(a)

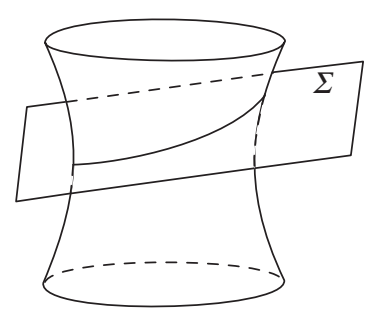

(b)

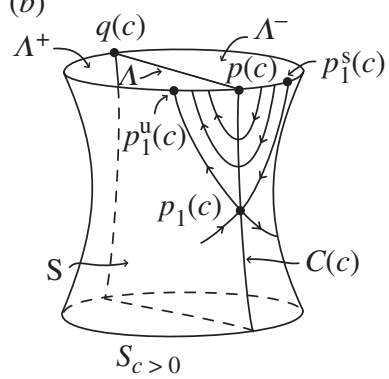

(c)

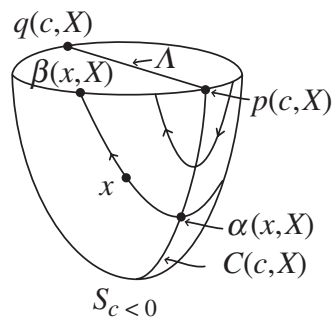

Figure 3. The construction of the topological equivalence when $f=x_{1}^{2}+x_{2}^{2}-x_{3}^{2}$.

in an appropriate coordinate system. The eigenvalues of $D X(0)$ are 0 and

$$
\pm \sqrt{\beta^{2}+\gamma^{2}-\alpha^{2}}
$$

Let

$$
\mathcal{B}=\left\{X \in V^{1}(\mathrm{~d} f): \beta^{2}+\gamma^{2}-\alpha^{2}>0\right\} .
$$

Let $S_{c}=f^{-1}(c)$ and $X \in \mathcal{B}$. Then $\left.X\right|_{S_{c>0}}$ has two singularities (both saddles) and these lie on a smooth curve $L$ tangent to the kernel of $D X(0)$ (Figure $2 a$ ). The vector field $\left.X\right|_{S_{c<0}}$ has no singularities. Let $\Sigma$ be a smooth surface containing the curve $L$, transverse to $S_{c>0}$ and such that $\Sigma \cap S_{c<0}=\emptyset$ and $\Sigma \cap S_{0}=\{0\}$ (Figure 3a). Write $X=\left(f_{1}, f_{2}, f_{3}\right)$ and let $S$ be the surface given by $f_{3}\left(x_{1}, x_{2}, x_{3}\right)=0$. Then $S$ is smooth (as $\beta=\partial f_{3} / \partial x_{1}(0) \neq 0$ or $\gamma=\partial f_{3} / \partial x_{2}(0) \neq 0$ ) and contains the singularities of $\left.X\right|_{S_{c}>0}$ (Figure $3 b$ ). Let $P$ be the plane $x_{3}=\bar{x}_{3}$, with $\bar{x}_{3}$ a positive constant close to zero. The vector field $X$ is transverse to $P \cap S_{c}$ except at two points denoted by $p(c)$ and $q(c)$ (Figure $3 c$ ).

The surface $S$ intersects $S_{c}$ on a curve $C$. Let $p_{1}(c)$ and $p_{2}(c)$ be the singularities of $\left.X\right|_{S_{c>0}}$, and denote by $p_{i}^{\mathrm{s}}(c)$ (respectively, $\left.p_{i}^{\mathrm{u}}(c)\right), i=1,2$, the intersection of the stable (respectively, unstable) manifold of $\left.X\right|_{S_{c>0}}$ with $P$. The set $\Lambda=P \cap S$ divides $P$ into two regions, $\Lambda^{-}$which contains $p_{1}^{\mathrm{s}}(c)$ and $p_{2}^{\mathrm{s}}(c)$ and $\Lambda^{+}$which contains $p_{1}^{\mathrm{u}}(c)$ and $p_{2}^{\mathrm{u}}(c)$; see Figure 3. (We add the letter $X$ to the above notation when necessary.)

Given $Y \in \mathcal{B}$, one can consider the same plane $P$ and construct the surfaces $\Sigma(Y)$, $S(Y)$ and the special curves, points and regions in a similar way as above. A topological equivalence between $X$ and $Y$, preserving the level sets of $f$, is constructed from a homeomorphism $h: P \rightarrow P$ satisfying the following properties:

- $h$ maps every circle $P \cap S_{c}$ to itself with the points $p(c, X), q(c, X), p_{i}^{\sigma}(c, X)$, $i=1,2, \sigma=\mathrm{u}, \mathrm{s}$, sent to their corresponding points for $Y$;

- the region $\Lambda^{-}(X)$ is mapped to $\Lambda^{-}(Y)$ in such a way that the $\operatorname{arcs}[p(c, X)$, $\left.p_{1}^{\mathrm{s}}(c, X)\right],\left[p_{1}(c, X)^{\mathrm{s}}, p_{2}^{\mathrm{s}}(c, X)\right]$ and $\left[p_{2}^{\mathrm{s}}(c, X), q(c, X)\right]$ are also sent to their corresponding arcs for $Y$;

- if $x \in \Lambda^{+}(X)$, let $\gamma(x, X)$ be the trajectory of $X$ through $x$ at $t=0$. Then $h(x)=$ $\gamma(h(a(x)), Y) \cap \Lambda^{+}(Y)$ if $a(x)=\gamma(x, X) \cap \Lambda^{-}(X) \neq \emptyset$, otherwise no condition is imposed on $h$ (i.e. it can be any homeomorphism if $\left.\gamma(x, X) \cap \Lambda^{-}(X)=\emptyset\right)$. 
The map $h$ is then extended as follows to the upper semi-space bounded by $\Sigma(X)$, with $h(\Sigma(X))=\Sigma(Y)$. If $c<0$ and $x \in S_{c}$, then the trajectory $\gamma(x, X)$ determines a point $\beta(x, X)$ on the plane $P$ and a point $\alpha(x, X)$ on the curve $C(c, X)$ such that $x$ is between $\alpha(x, X)$ and $\beta(x, X)$ (Figure $3 c$ ). The point $h(x)$ is defined as the unique point on $S_{c}$ such that $h(\beta(x, X))=\beta(h(x), Y)$ and

$$
\frac{c_{Y}[h(\beta(x, X)), h(x)]}{c_{Y}[h(\beta(x, X)), \alpha(h(x), Y)]}=\frac{c_{X}[\beta(x, X), x]}{c_{X}[\beta(x, X), \alpha(x, Y)]}
$$

(where $c_{Z}[p, q]$ denotes the length of the arc of the trajectory of a vector field $Z$ delimited by the points $p$ and $q$ ). The extension of the map $h$ is defined similarly on $S_{c \geqslant 0}$ by sliding along integral curves and using the definition of $h$ on the plane $P$. The same approach is used to define $h$ on the lower semi-space delimited by $\Sigma(X)$.

The above construction works for any $X, Y \in \mathcal{B}$. If $Y \in V^{1}(\mathrm{~d} f)$ is $C^{1}$-close to $X$, then the constants $\alpha^{\prime}, \beta^{\prime}, \gamma^{\prime}$ associated to $Y$ are sufficiently close to those associated to $X$. So $Y \in \mathcal{B}$ and therefore $(\mathrm{d} f, Y)$ is equivalent to $(\mathrm{d} f, X)$.

(ii) When $\beta^{2}+\gamma^{2}-\alpha^{2}<0$ the eigenvalues of $D X(0)$ are of the form $\lambda_{1}=0, \lambda_{2}=i \lambda$ and $\lambda_{3}=-i \lambda$. Following the arguments in case (a), there is a diffeomorphism $\phi$ such that $\phi_{*} X=\lambda T+X_{2}+R_{2}$ with $T, X_{2}, R_{2}$ as in case (a). Let

$$
\mathcal{C}=\left\{X \in V^{2}(\mathrm{~d} f): \beta^{2}+\gamma^{2}-\alpha^{2}<0, b \neq 0\right\} .
$$

When $b \neq 0, X$ has two singularities on $S_{c<0}$, one is an attractor and the other a repulsor. The type is completely determined by the sign of $b$. Given $Y \in \mathcal{C}$ with the associated constants $b^{\prime}$ having the same sign as $b$, Lara constructed a homeomorphism that conjugates $X$ and $Y$ while preserving the foliation of $\mathrm{d} f$ by combining the approaches in the proofs of the cases (a) and (i). If $Y \in V^{2}(\mathrm{~d} f)$ is $C^{2}$-close to $X \in \mathcal{C}$, then $Y \in \mathcal{C}$ and $b^{\prime}$ has the same sign as $b$, so $(\mathrm{d} f, Y)$ is equivalent to $(\mathrm{d} f, X)$.

Remark A 3. In his thesis, Lara also treated the case $n=4$ with $f=\sum_{i=1}^{4} \pm x_{i}^{2}$ and $n \geqslant 5$ with $f=\sum_{i=1}^{n} x_{i}^{2}$. He proved results on the topological stability of vector fields in $V^{3}(\mathrm{~d} f)$ in both cases.

\section{References}

1. C. Camacho And A. Lins Neto, Geometric theory of foliations (Birkhäuser, Boston, MA, 1985).

2. S.-N. CHOW, C. LI AND D. WANG, Normal forms and bifurcation of planar vector fields (Cambridge University Press, 1994).

3. A. S. DE MedeIRos, Topological stability of integrable differential forms, Springer Lecture Notes in Mathematics, Volume 597, pp. 197-225 (Springer, 1977).

4. G. A. LARA LunA, Estudo local dos campos vetoriais com uma integral primeira de Morse, Doctoral thesis, IMPA, Rio de Janeiro (1978).

5. H. Matsumura, Commutative ring theory, Cambridge Studies in Advanced Mathematics, Volume 8 (Cambridge University Press, 1986).

6. R. S. MoL, Flags of holomorphic foliations in $\mathbb{P}_{\mathbb{C}}^{3}$, preprint (2006). 
7. R. Moussu, Existence d'intégrales premières pour un germe de forme de Pfaff $C^{\infty}$, non dégénéré, Bol. Soc. Bras. Mat. 7 (1976), 111-120.

8. G. Reeb, Sur certain propriétés topologiques des variétés feuilletées, Publ. Inst. Math. Univ. Strasbourg 11 (1952), 5-89, 155-156.

9. J. Sотомаyor, Generic one-parameter families of vector fields on two-dimensional manifolds, Publ. Math. IHES 43 (1974), 5-46.

10. F. TAKens, Singularities of vector fields, Publ. Math. IHES 43 (1974), 47-100.

11. C. T. C. WALl, Finite determinacy of smooth map-germs, Bull. Lond. Math. Soc. 13 (1981), 481-539.

12. M. Zhitomirskin, Typical singularities of differential 1-forms and Pfaffian equations, Translations of Mathematical Monographs, Volume 113 (American Mathematical Society, Providence, RI, 1992). 